\title{
Phosphorylation-dependent neurofilament epitopes are reduced at the node of Ranvier
}

\author{
M. MATA*, N. KUPINA and D. J. FINK \\ Department of Neurology, University of Michigan and GRECC Neurology Research Laboratory, VAMC, Ann Arbor, MI, USA
}

Received 7 June 1991; revised 8 August and 20 September 1991; accepted 1 October 1991

\section{Summary}

Neurofilaments in axons are highly phosphorylated at multiple sites on the $200 \mathrm{kDa}$ neurofilament (neurofilament-H) and $160 \mathrm{kDa}$ (neurofilament-M) subunit peptides. We used a panel of monoclonal and polyclonal antibodies against distinct neurofilament epitopes to study the distribution of these epitopes along the axons of large myelinated fibres in rat sciatic nerve using quantitative electron microscopic immunocytochemistry with colloidal gold. Antibodies specific for phosphorylated epitopes on neurofilament-H showed a $60 \%$ reduction in density of immunoreactivity at the node of Ranvier, compared to the internodal axon. Antibodies directed against neurofilament-M, which recognized phosphorylated epitopes preferentially, showed a $40 \%$ reduction in density of immunoreactivity at the node. Following dephosphorylation of the neurofilaments in tissue sections by alkaline phosphatase treatment, antibodies which recognized dephosphorylated forms of neurofilament- $\mathrm{H}$ showed no reduction in density of immunoreactivity at the node. Similarly, an antibody directed against the $70 \mathrm{kDa}$ subunit (neurofilament-L), showed no reduction in density of immunoreactivity at the node.

An antibody against tubulin also showed no decrease in the density of immunoreactivity at the node of Ranvier. Tubulin immunoreactivity was similar in myelinated and unmyelinated fibres. In contrast to phosphorylated neurofilament epitopes, immunoreactivity was much greater in myelinated than unmyelinated fibres.

These results suggest that the degree of phosphorylation of neurofilament- $\mathrm{H}$ and neurofilament- $\mathrm{M}$ subunits is reduced at the node of Ranvier, in comparison to internodal neurofilaments, and imply that a post-translational modification of neurofilaments must occur along the length of the axon at the node.

\section{Introduction}

The principal cytoskeletal element of large myelinated axons are neurofilaments (NFs), which in mammals are composed of three subunits with apparent molecular weight of $70 \mathrm{kDa}(\mathrm{NF}-\mathrm{L}), 160 \mathrm{kDa}(\mathrm{NF}-\mathrm{M})$ and $200 \mathrm{kDa}$ (NF-H) (Hoffman \& Lasek, 1975). The individual NF protein subunits are highly phosphorylated, principally in repeat sequences in the carboxy terminals of the NF-H and NF-M subunits (Julien \& Mushynski, 1982, 1983). Several laboratories have developed specific monoclonal antibodies which recognize either phosphorylated or non-phosphorylated epitopes of NF proteins (Sternberger \& Sternberger, 1983; Lee et al., 1987). Neurofilaments appear to be phosphorylated upon or soon after entry into axons, so that those neurofilaments in the cell body are not phosphorylated, while those in the axons in normal conditions appear to be highly phosphorylated (Bennett \& DiLullo, 1985; Hart et al., 1987; Lee et al., 1987; Pestronk et al., 1990).

Although phosphorylated NFs are found through-

out the length of the axon, NF phosphorylation and dephosphorylation is a dynamic process within the axon. Several different neurofilament kinases have been identified (Julien et al., 1983; Sihag \& Nixon, 1989; Dosemeci et al., 1990), and there is indirect evidence that the aggregate phosphorylation state of NFs differs in different regions of the axon (Nixon \& Lewis, 1986; Nixon et al., 1987). It has been proposed that phosphorylation of NF subunits may affect the assembly of NF subunit peptides into neurofilaments (Lewis \& Nixon, 1988; Gonda et al., 1990), the stability of formed NFs (Wong et al., 1984; Pant, 1988), the interaction of NFs with microtubules (Minami \& Sakai, 1985; Hisanaga \& Hirokawa, 1990), or with other NFs, although recent evidence suggests that dephosphorylation in vitro may not affect some of these processes (Wong et al., 1984; Hisanaga \& Hirokawa, 1989).

Neurofilaments play a critical role in defining the structure of the axon of large myelinated fibres (Friede \& Samorajski, 1970; Hoffman et al., 1984, 1985), and

* To whom correspondence should be addressed at: Department of Neurology, 2215 Fuller Road, Ann Arbor MI 48105, USA. 
alterations in NF organization commonly characterize disorders leading to axonal degeneration (Griffin et al., 1983; Parhad et al., 1984; Watson \& Griffin, 1987, 1988), and may be associated with alterations in the phosphorylation state of axonal NFs (Rosenfeld et al., 1987; Gold et al., 1988). These pathologic processes often occur multifocally within axons, and therefore it is important to define, in large myelinated fibres, whether there is discrete regional regulation of NF phosphorylation. We used commercially available monoclonal and polyclonal antibodies raised against NF subunits and quantitative electron microscopic immunocytochemistry with colloidal gold to determine the ultrastructural distribution of specific neurofilament epitopes in axons. We show that at the node of Ranvier, there is a reduction in the degree of NF phosphorylation compared to the internodal axon, defining an ultrastructural heterogeneity along individual myelinated axons.

\section{Materials and methods}

\section{Antibodies}

We used ten antibodies which proved to be useful in electron microscopic immunocytochemistry on LR White sections. Antibodies against NF-H included rabbit polyclonal AB1982 (Chemicon), mouse monoclonal antibodies SMI-31 and SMI-32 (Sternberger-Meyer Immunocytochemicals, Inc.) and N-52 and NE-14 (Boehringer-Mannheim Biochemicals). Antibodies predominantly recognizing NF-M were rabbit polyclonal AB1981 (Chemicon) and mouse monoclonal NN-18 (Boehringer-Mannheim). Antibodies specific for NF-L were rabbit polyclonal AB1983 (Chemicon) and mouse monoclonal NR-4 (Boehringer-Mannheim). In addition, we used a rabbit polyclonal antitubulin antibody BT-578 (Biomedical Technologies, Inc).

\section{Immunocytochemistry}

Male Sprague Dawley rats, weighing 200-300 g, were perfused through the heart with $100 \mathrm{mM}$ phosphate buffer followed by a mixture of $0.5 \%$ glutaraldehyde, $4 \%$ paraformaldehyde or $4 \%$ paraformaldehyde alone in phosphate buffer with $2.5 \mathrm{~mm} \mathrm{MgCl}$. The sciatic nerve was removed, placed in fresh fixative solution an additional $2 \mathrm{~h}$, and then embedded in LR White.

Ultrathin sections were exposed to the antisera for $2 \mathrm{~h}$ at room temperature, followed by the appropriate secondary antiserum bound to $15 \mathrm{~nm}$ colloidal gold (E-Y Laboratories), at room temperature, for $1 \mathrm{~h}$. The grids were stained for 20 min with uranyl acetate and examined in a JEOL 100-S electron microscope.

As a control for the immunocytochemical reaction, we carried out identical procedures but deleted the primary antibody.

\section{Dephosphorylation of neurofilaments for electron microscopy}

In order to assess the density of NF immunoreactivity independent of phosphorylation state, NFs were dephos- phorylated in situ using alkaline phosphatase. We used two different methods to dephosphorylate NFs in situ with alkaline phosphatase. In the first method, $4 \%$ paraformaldehyde fixed sciatic nerve was dissected into longitudinal sections containing a few sections each and incubated overnight with $1.2 \mathrm{U}$ of alkaline phosphatase from $E$. coli (Sigma P 4252) in $0.1 \mathrm{M}$ Tris, $\mathrm{pH} \mathrm{8.1.} \mathrm{The} \mathrm{tissue} \mathrm{was} \mathrm{then}$ rinsed, dehydrated and embedded in LR White for EM immunocytochemistry. In the second method, EM sections of $4 \%$ paraformaldehyde fixed nerve were incubated overnight at $37^{\circ} \mathrm{C}$ on a $40 \mathrm{ml}$ drop containing $1.2 \mathrm{U}$ of alkaline phosphatase, rinsed, and processed for EM immunocytochemistry.
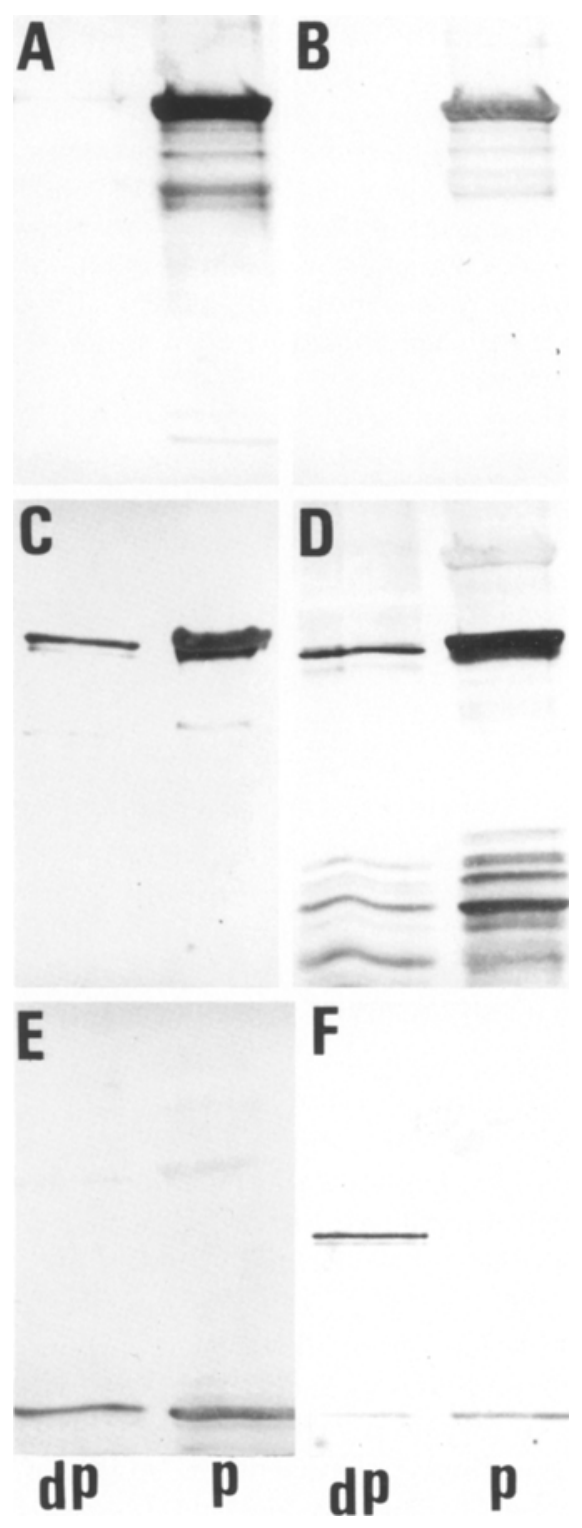

Fig. 1. Western blot of isolated NFs (p) and alkaline phosphatase treated NFs (dp) using the following antibodies: (A) SMI-31, 1:500; (B) NE-14, 1:100; (C) NN-18, $1: 100 ;$ (D) AB1981, 1:500; (E) AB1983, 1:500; (F) N-52, 1:250. 

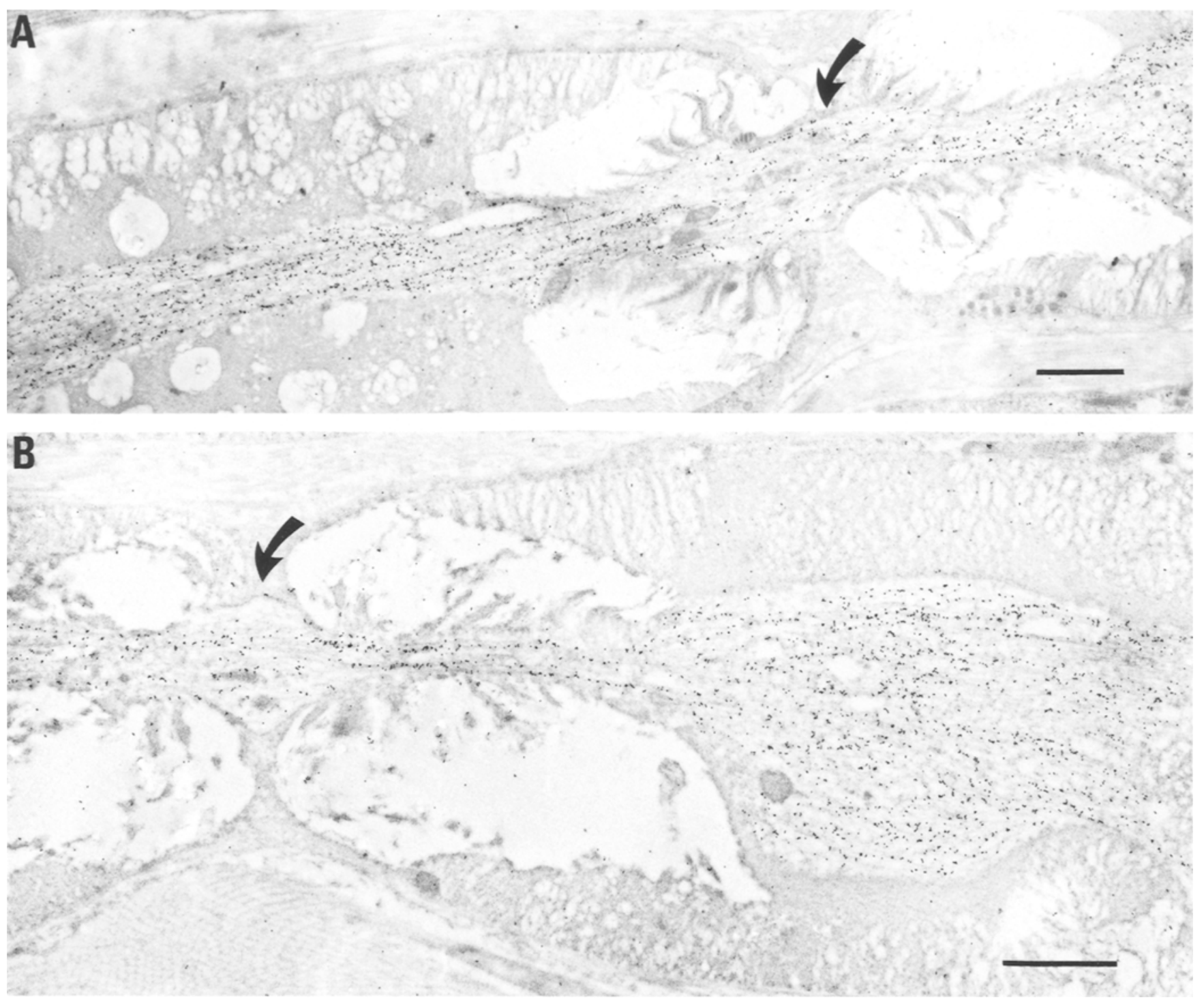

Fig. 2. Electron-microscopic immunocytochemistry of sciatic nerve with SMI-31 (specific for phosphorylated NF-H). The node of Ranvier is indicated by the curved arrow. Primary antibody $1: 150$. Scale bar $=1 \mu \mathrm{m}$.

\section{Quantitative analysis of immunoreactivity}

In order to obtain a quantitative estimate of the relationship of gold particle density at the node to the density of gold particles in the internode, the grids were photographed and printed at $\times 13500$. The density of gold particles per $\mathrm{cm}^{2}$ at the node and at least two different regions of the internode was determined with an automated image analyzer (Imaging Resources Inc., London, Ontario), as previously described (Mata et al., 1990), by determining the proportional area of gold grains in defined areas within the node and internode respectively. These usually included the entire nodal axoplasm, and selected representative areas of internodal axoplasm. The node was strictly defined as the region between the attachment sites of the paranodal loops of the Schwann cell, and care was taken to assess the internodal axon distant from the paranodal region beneath the paranodal loops. The density of gold grains in the node was compared to the density in the internode for each fibre examined, and a ratio of node/internode gold grain density determined. This ratio, determined for each individual nerve fibre with a single antibody, could then be compared using different antibodies on the same or different fibres. The statistical significance of the difference between ratios obtained with different antibodies was determined by a one-way analysis of variance with multiple post hoc pairwise comparisons using the Tukey HSD post hoc test (Wilkerson, 1990).

\section{Isolation of neurofilaments}

Neurofilaments were isolated from sciatic nerve using the method described by Schlaepfer as modified by Mori and Kurokawa (1980). The rats were killed by decapitation, the sciatic nerves removed, washed with saline, and homogenized with a Polytron in 2 volumes of $10 \mathrm{~mm}$ phosphate buffer (pH 7.5) containing $5 \mathrm{~mm}$ EDTA. The homogenate was kept at $4^{\circ} \mathrm{C}$ for $60 \mathrm{~min}$ and then centrifuged at $\times 20000 \mathrm{~g}$ for 30 

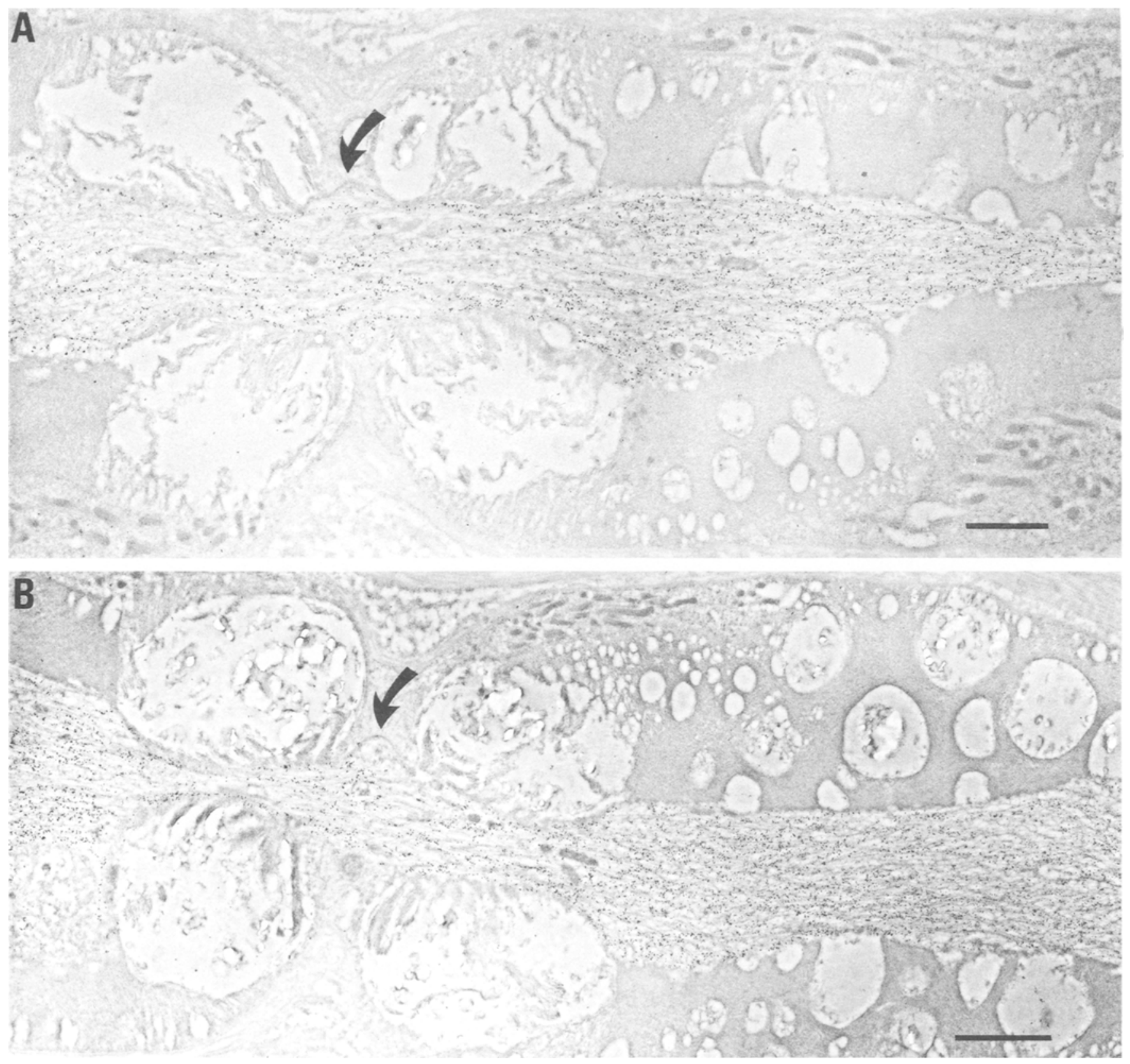

Fig. 3. Electron-microscopic immunocytochemistry of sciatic nerve with NE-14 (specific for phosphorylated NF-H). Node, curved arrow. Primary antibody 1:50. Scale bar $=1 \mu \mathrm{m}$.

min at $0^{\circ} \mathrm{C}$. The supernatant was adjusted to $0.1 \mathrm{M} \mathrm{NaCl}$ and then centrifuged again. The resulting supernatant was layered on top of $1 \mathrm{~m}$ sucrose in $10 \mathrm{~mm}$ phosphate buffer, $\mathrm{pH}$ $7.5,0.1 \mathrm{M} \mathrm{NaCl}, 5 \mathrm{~mm}$ EDTA, and centrifuged at $\times 130000 \mathrm{~g}$ for $2 \mathrm{~h}$ at $4^{\circ} \mathrm{C}$. The NFs were recovered as a pellet and resuspended in $0.1 \mathrm{M}$ Tris, $\mathrm{pH} 6.8$.

\section{Dephosphorylation of isolated neurofilaments}

In order to test the reactivity of the antibodies against dephosphorylated NFs, the isolated NFs were dephosphorylated with alkaline phosphatase. Twenty-five micrograms of NFs prepared as described were dialyzed overnight at $37^{\circ} \mathrm{C}$ with $3 \mathrm{U}$ alkaline phosphatatse (Sigma) against a buffer containing $100 \mathrm{~mm}$ Tris, pH 6.8, with 5 mM EDTA.

\section{Western blot}

In order to determine the specificity of each of the antisera, $2 \mathrm{mg}$ of native NFs isolated from sciatic nerve and $2 \mathrm{mg}$ of sciatic nerve NFs dephosphorylated by alkaline phosphatase were run in adjacent lanes of a $7.5 \%$ SDS-polyacrylamide gel and transferred to nitrocellulose in Towbin buffer (Towbin et al., 1979). The blot was reacted with the primary antibody followed by peroxidase-conjugated secondary 


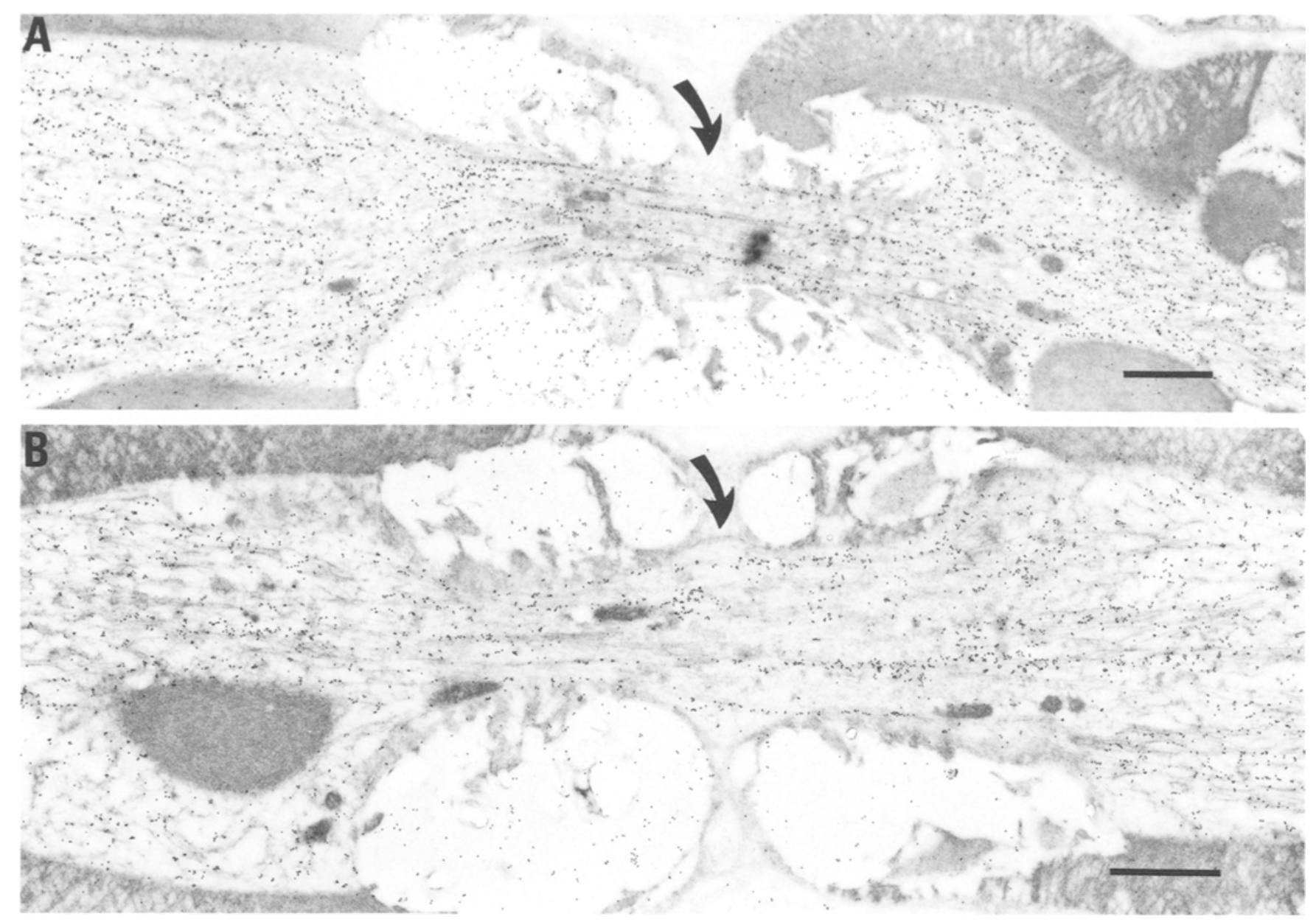

Fig. 4. EM immunocytochemistry of sciatic nerve with AB1981 (specific for NF-M, phosphorylation dependent). Node, curved arrow. Primary antibody AB1981 1:50. Scale bar $=1 \mu \mathrm{m}$.

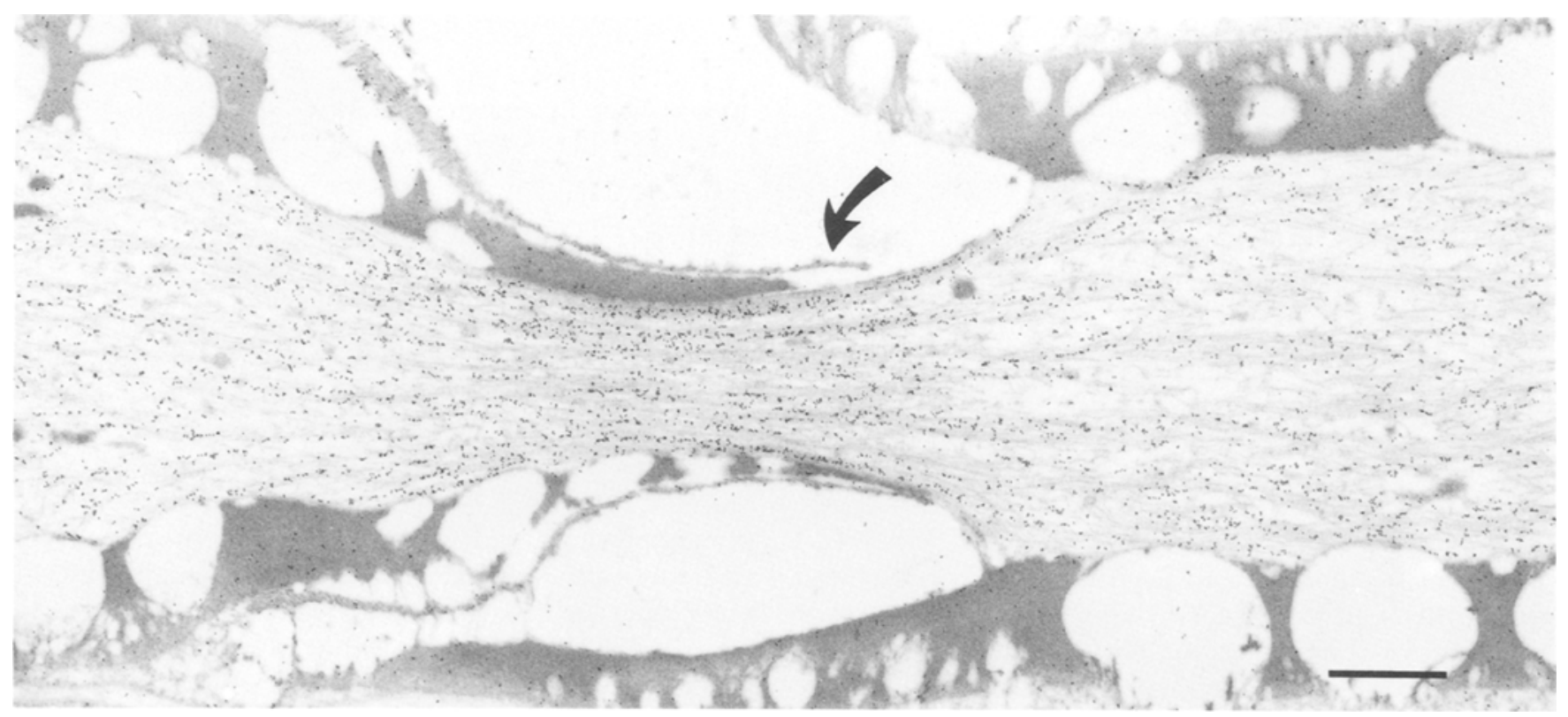

Fig. 5. Artifactual constriction of internodal axon immunostained with SMI-31 (specific for phosphorylated NF-H) shows an increase in density of immunoreactivity at the site of the constriction (arrow). Primary antibody $1: 100$. Scale bar $=1 \mu \mathrm{m}$. 

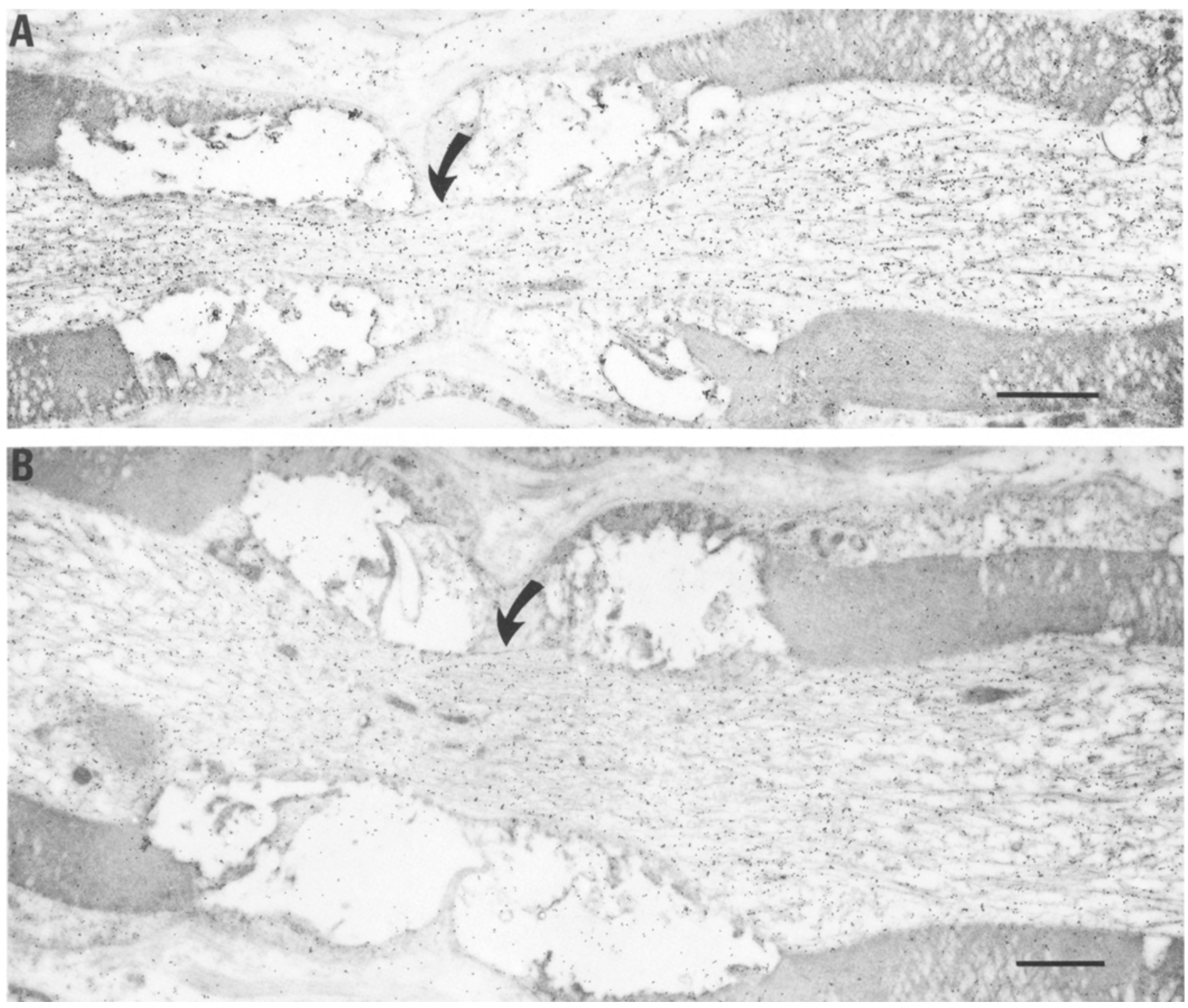

Fig. 6. Sciatic nerve stained with AB1983 (specific for NF-L, phosphorylation independent). Primary antibody 1:50. Scale bar $=1 \mu \mathrm{m}$.

antibody $(1: 250$ to $1: 2500)$ and developed with 4 chloro-1naphthol as described by Siegel and colleagues (1986).

\section{Results}

The antibodies used in this study have all been previously characterized (Sternberger \& Sternberger, 1983; Shaw et al., 1986; Karlsson et al., 1989). However, in order to determine the specificity of the antibodies for proteins in the tissue which we were studying immunocytochemically, we performed Western blot of native and alkaline phosphatase treated NFs isolated from sciatic nerve ( $\mathrm{P}$ and $\mathrm{dP}$ in Fig. 1). None of the antibodies were absolutely specific for a single band, but each showed a pattern of immunoreactivity that was sufficiently distinctive. SMI-31 (A) and NE-14 (B) recognized predominantly NF-H in its native but not dephosphorylated form. Minor amounts of immunoreactive lower molecular weight bands, presumably breakdown products, and NF-M were also seen on these densely reacted blots, greater for SMI-31 than for NE-14. NN-18 (C) and AB1981 (D) reacted predominantly with NF-M in its native form. Alkaline phosphatase treated NFs were still immunoreactive with these antibodies, but the amount of immunoreactivity was substantially reduced by dephosphorylation. AB1983 (E) and NR-4 (not shown) were specific for NF-L, and reacted similarly with native and dephosphorylated NFs, in agreement with previous data showing that NF-L is not phosphorylated in nerve. N-52 (F) and SMI-32 (not shown) (Sternberger 


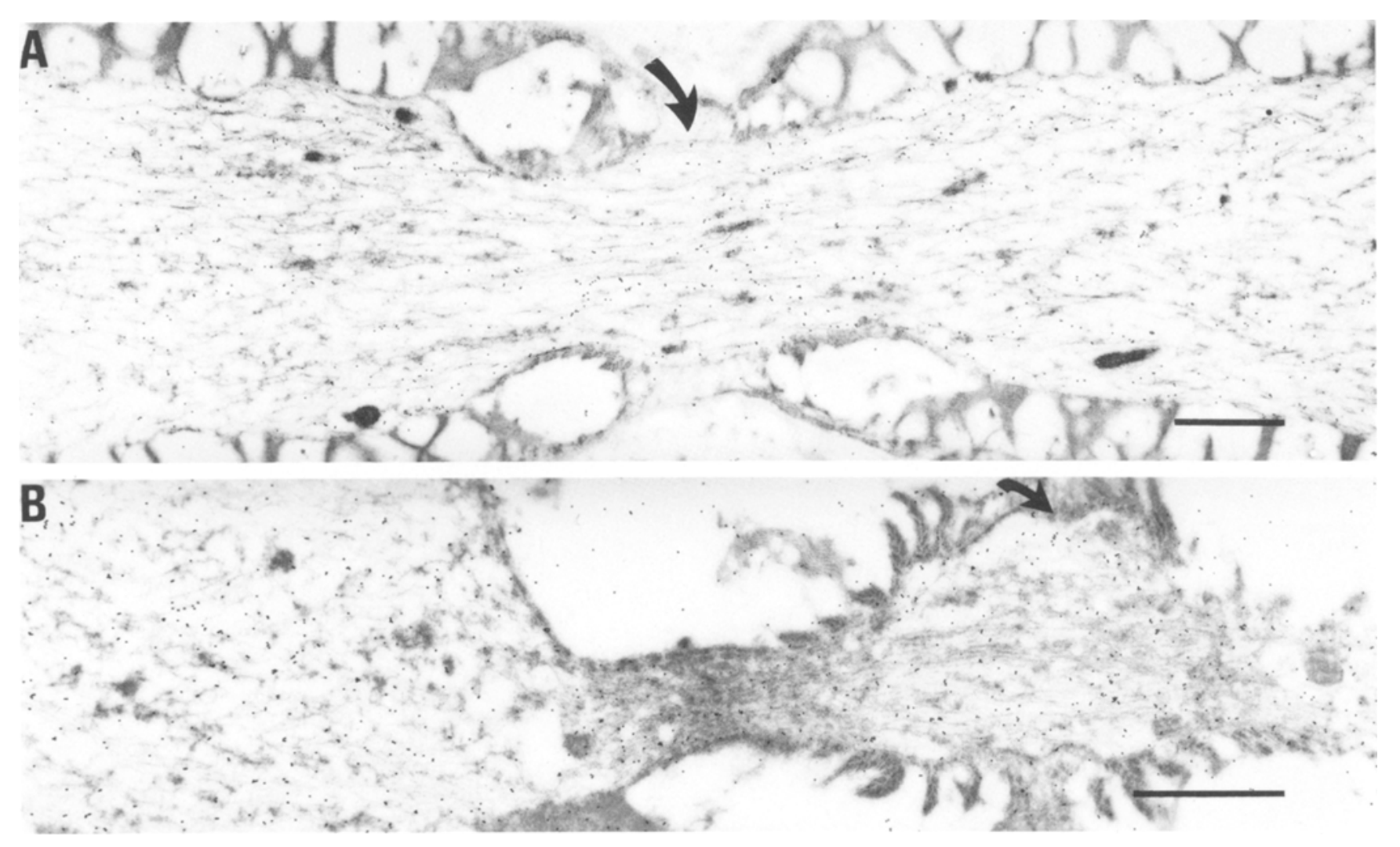

Fig. 7. Distribution of NF immunoreactivity in axons dephosphorylated with alkaline phosphatase. (A) N-52, (specific for dephosphorylated NF-H). Dephosphorylation of thin sections on grid as described in methods. (B) SMI-32, (specific for dephosphorylated NF-H). Dephosphorylation of tissue before embedding. Scale bar $=1 \mu \mathrm{m}$.

\& Sternberger, 1983) reacted with dephosphorylated NF-H but not with native phosphorylated forms of NF. N-52 cross reacted to a small degree with both native and alkaline phosphatase treated NF-L. SMI-32 did not cross react with NF-L.

Electron microscopic immunocytochemistry with antibodies specific for phosphorylated NF-H, SMI-31 (Fig. 2), and NE-14 (Fig. 3), showed that the density of immunoreactivity at the node of Ranvier (curved arrows) was markedly reduced compared to the density of immunoreactivity at the internode. Along stretches of internode, the immunoreactivity appeared to be of a constant density (data not shown), and that density was the same in the central region of the internode as it was in the internodal axoplasm near but not including the paranode. With antibodies specific for NF-M (Fig. 4) a similar reduction in the density of immunoreactivity was also seen at nodes of Ranvier.

The node of Ranvier in large myelinated fibres is narrower than the internodal axon. However, the decrease in density of immunoreactivity at the node using antibodies to NF-H and NF-M was not an artifact of the axonal constriction. In isolated instances internodal axons could be found which appeared constricted in the region beneath the Schmidt Lanterman cleft as a result of fixation artifact. In these circumstances, the density of immunoreactivity uniformly increased, in accordance with the compression of NFs in that region (Fig. 5).

Grids stained with antibodies directed against NF-L (Fig. 6) showed no reduction in the density of immunoreactivity at the node compared to the internode. Antibodies directed against nonphosphorylated NFs (SMI32 and N-52) showed no immunoreactivity above background on normal nerve. However, grids in which the NFs were dephosphorylated with alkaline phosphatase prior to immunostaining with antibodies reactive with dephosphorylated epitopes (N-52 and SMI-32) showed a density of immunoreactivity at the node that was similar to the internode (Fig. 7). The tissue dephosphorylated prior to embedding showed no immunoreactivity to phosphorylated epitopes using NE-14. These data suggest that the alkaline phosphatase treatment of the tissue fully dephosphorylated the NFs, and that the NF protein was present in equal density at the node and the internode.

Using the antibody to tubulin, it was apparent that density of immunostaining at the node was, if anything, greater than the density of immunoreactivity in the internode (Fig. 8), in contrast to the reduction seen 


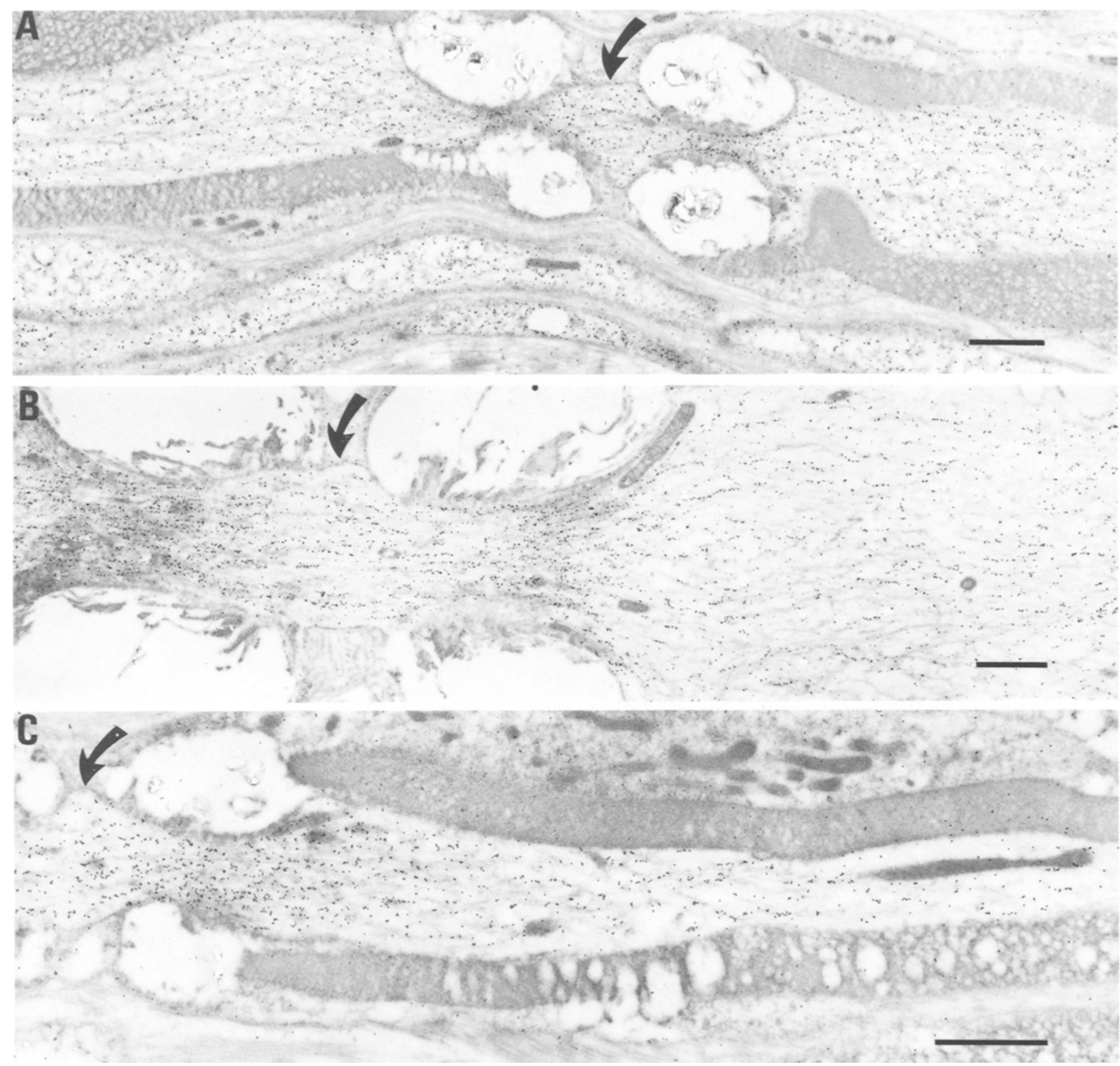

Fig. 8. Tubulin immunoreactivity at the node of Ranvier. Primary antibody BT 578, 1:100. Scale bar $=1 \mu \mathrm{m}$.

with anti-NF-H and anti-NF-M antibodies. This finding adds support to the conclusion that the reduction in density of immunoreactivity with the NF antibodies was not artifactual. With the anti-tubulin antibody the density of immunoreactivity in myelinated fibres was similar to that in unmyelinated fibres. Neurofilament immunoreactivity was not detectable in unmyelinated fibres (Fig. 9). This is in agreement with the known distribution of NFs and microtubules in myelinated and unmyelinated fibres respectively (Berthold, 1978). The density of immunoreactivity using antibodies which recognize phosphorylated epitopes appeared to increase with fibre diameter, although that relationship was not evaluated quantitatively. Control immunostaining with deletion of the primary antibody showed the absence of immunoreactivity (Fig. 10).

The density of immunoreactivity was measured quantitatively with an image analyzer, and the results expressed as the ratio of nodal to internodal immunoreactivity (Fig. 11). For NF-L the ratio of nodal to internodal immunoreactivity was 1 . With the anti-NF$\mathrm{H}$ and anti-NF-P antibodies the density of immuno- 

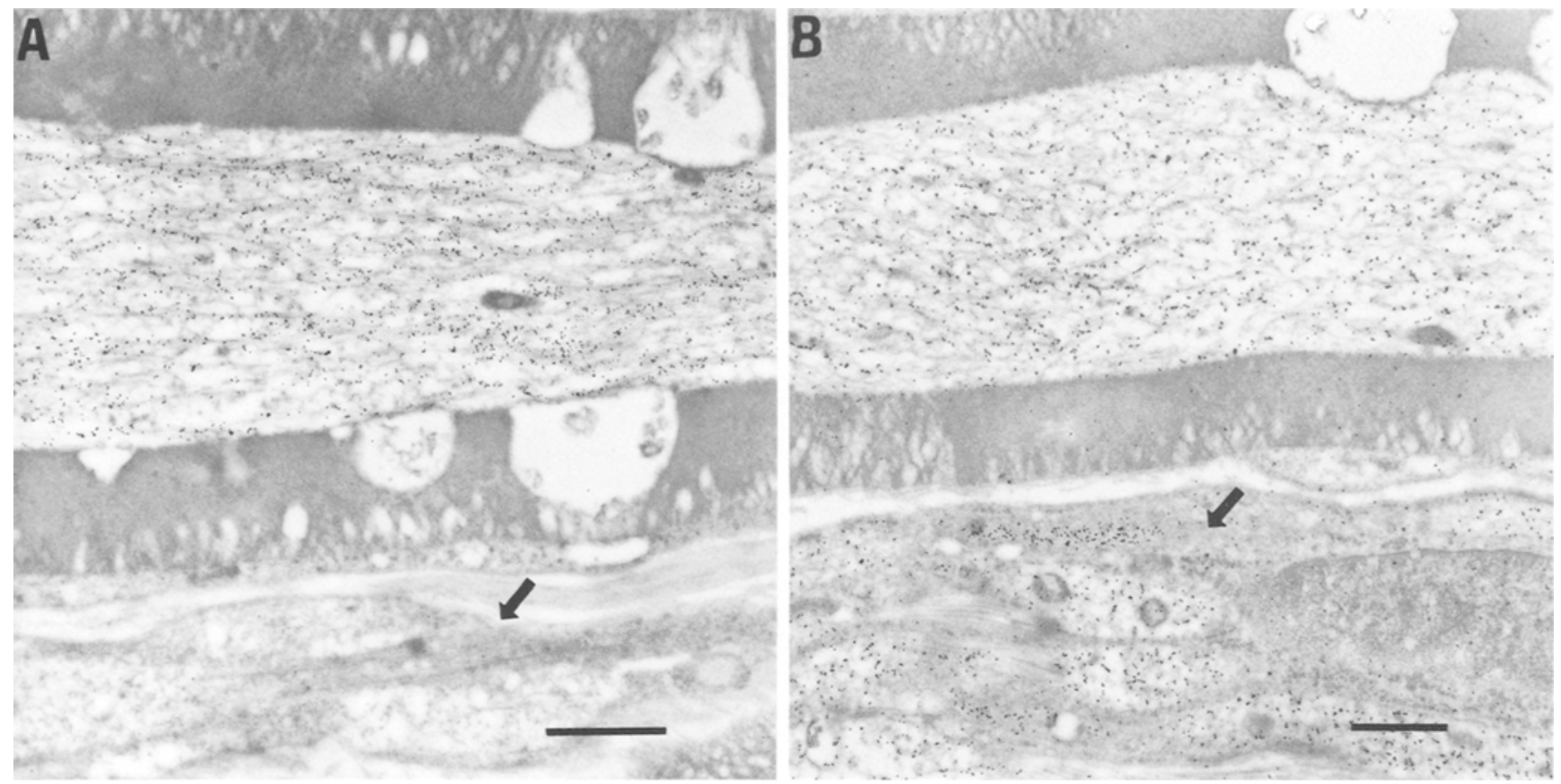

Fig. 9. (A) NF (SMI-31) and (B) tubulin (BT578) immunoreactivity in myelinated and unmyelinated fibres of sciatic nerve. Arrow points to unmyelinated fibres. Both antibodies $1: 100$. Scale bar $=1 \mu \mathrm{m}$.

reactivity (Fig. 11). For NF-L the ratio of nodal to internodal immunoreactivity was 1 . With the anti-NF$\mathrm{H}$ and anti-NF-P antibodies the density of immunoreactivity at the node was reduced by $60 \%$ compared to the internodal density. Immunoreactivity of NF-M was reduced by $40 \%$ at the node, compared to the internode. This may correlate with the fact that the anti NF-M antibodies employed cross-react with dephosphorylated forms. The ratio of density of immunoreactivity at the node to density of immunoreactivity at the internode on alkaline phosphatase treated grids stained with antibodies SMI-32 or N-52, was 1 . In comparison, the ratio of density of immunoreactivity at the node of density of immunoreactivity at the internode using the anti-tubulin antibody was 1.8.

\section{Discussion}

The principal finding of this study is that NFs, which are highly phosphorylated in myelinated axons, do not appear to be phosphorylated uniformly along the length of individual fibres. Using antibodies which recognize phosphorylation-dependent epitopes on $\mathrm{NF}-\mathrm{H}$ and NF-M, there is a reduction in the density of immunoreactivity at the node of Ranvier. In contrast, an antibody to NF-L which is relatively insensitive to the phosphorylation state of NF-L, shows no reduction in density of immunoreactivity at the node. We used tissue embedded in LR White because this medium most effectively preserves immunoreactivity for electron microscopy (Mata et al., 1990). An un- avoidable consequence of this choice is the poor preservation of myelin, but this is not of consequence in the present study. However, the cytoskeleton was well preserved with this procedure, and linearly oriented neurofilaments within the axoplasm were demonstrated on sections from osmicated tissue. It is not possible, in tissue embedded in LR White, to count the density of unstained NFs at the node. However, in as previous careful study of large myelinated fibres in the sciatic nerve of the cat, Berthold (1978) showed that NFs appear to have a fixed spacing and uniform density along the length of the fibre. The number of NFs at the constriction at the node is reduced compared to the internodal axon, but the reduction is proportional to the reduced cross sectional area, so that NF density remains constant. In contrast, he found that the density of microtubules at the constriction at the node of Ranvier is increased compared to the internodal axon (Berthold, 1978). The quantitative immunocytochemical data reported here for NF-L, for alkaline phosphatase dephosphorylated NF-H, and for tubulin (both with single fibres and for the comparison of myelinated to unmyelinated fibres) are in accord with the previously reported morphometric study. The concordance of quantitative immunocytochemistry with morphometry serves as one validation of the quantitative immunocy tochemical technique.

The reduction of immunoreactivity at the node of Ranvier seen with antibodies that preferentially recognize phosphorylation-dependent epitopes, demonstrates that a post-translational modification takes 

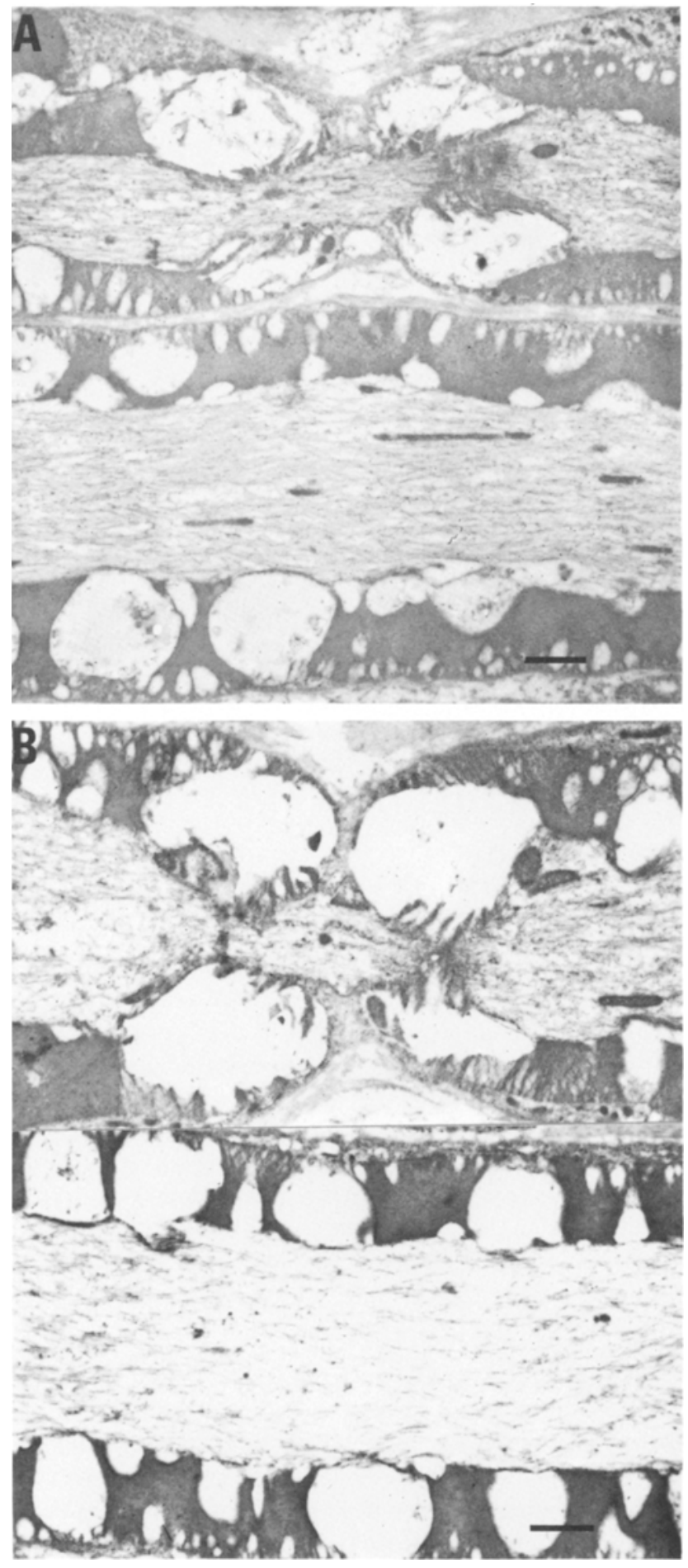

Fig. 10. Controls with deletion of the primary antibody. (A) Goat anti-mouse secondary antibody; (B) goat anti-rabbit secondary antibody. Scale bar $=1 \mu \mathrm{m}$.

place at the node of Ranvier. The most likely modification is partial dephosphorylation, resulting in the removal of phosphate groups critical to antibody recognition. The NFs are not completely dephosphorylated at the node, because the antibodies specific

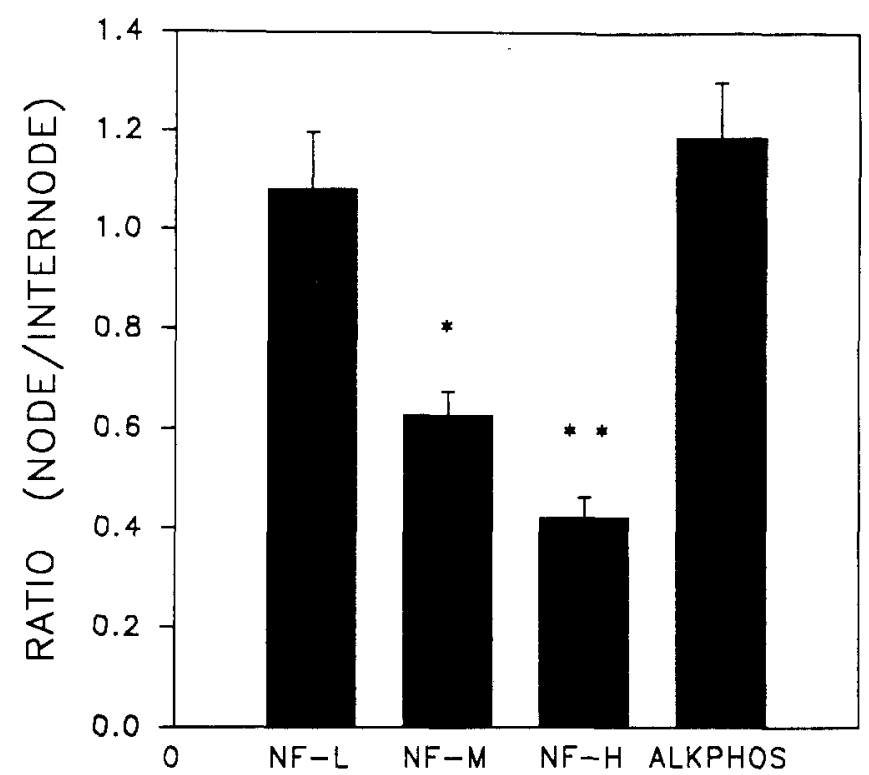

Fig. 11. The ratio of the density of immunoreactivity of the node to the density of immunoreactivity at the internode in individual fibres was calculated using automated image analysis. The antibodies used were NF-L (phosphorylation independent): AB1983, and NR-4 (19 nodes); NF-M (phosphorylation dependent): AB1981 and NN-18 (19 nodes); NF-H (specific for phosphorylated NF-H): SMI-31 and NE-14 (38 nodes). The column labeled Alkphos represents the ratio of immunoreactivity (node/internode) of thin sections dephosphorylated with alkaline phosphatase ( 25 nodes) and reacted with antibodies that recognize dephosphorylated NF-H (SMI-32 and N-52). Mean \pm standard deviation, ${ }^{*} \mathrm{P}<.01,{ }^{* *} \mathrm{P}<.001$, compared to NF-L.

for fully dephosphorylated NFs (SMI-32 and N-52) do not label either the internodal axon or the node in untreated grids. An alternative explanation is that NFs at the node have a higher ratio of NF-L to the other subunits, similar to late appearance of NF-H in development (Shaw \& Weber 1982; Willard \& Simon 1983). The experiments with alkaline phosphatase treated grids suggest that this is not the case. Thus, like NF-L, the density of the protein backbone appears similar in the two locations. Finally, it is also possible that some other post-translational modification at the node might obscure the epitope recognized by the phosphorylation-dependent antibodies. However, unless alkaline phosphatase treatment reversed this putative modification, the alkaline phosphatase experiments would argue against this interpretation.

The functional significance of NF phosphorylation is not known. Neurofilaments in neuronal perikarya are predominantly non-phosphorylated (Sternberger \& Sternberger, 1983). Phosphorylation takes place soon after NFs enter axons, but there is biochemical evidence for continuous phosphate turnover on neurofilament subunits during axonal transport (Nixon \& Lewis, 1986). It has been proposed that the 
degree of NF phosphorylation is greater in longer axons (Sternberger et al., 1982), although recent data comparing different CNS tracts of similar length suggest that phosphorylation state may correlate better with differences in NF density than with axonal length (Szaro et al., 1990). It has been suggested that phosphorylation may alter the interaction of NFs with microtubules, although experiments in vitro have demonstrated both that NF dephosphorylation may suppress microtubule polymerization and that dephosphorylated NFs preferentially bind to polymerized microtubules (Minami \& Sakai, 1985; Hisanaga \& Hirokawa, 1990). It has also been suggested that phosphorylation of NF subunits may correlate with slower net rates of axonal transport of NFs (Lewis \& Nixon, 1989; Watson et al., 1990). And there is other evidence from in vitro studies, that phosphorylation may protect NFs from proteolysis (Goldstein et al.,

\section{References}

BENNET, G. S. \& Dilullo, C. (1985) Slow posttranslational modification of a neurofilament protein. Journal of Cell Biology 100, 1799-1804.

BERTHOLD, C.-H. (1978) Morphology of normal peripheral axons. In Physiology and Pathobiology of Axons, (edited by WAXMAN, S. G.) pp. 3-64. New York: Raven Press.

DosemeCI, A., FLOYD, C. C. \& PANT, H. C. (1990) Characterization of neurofilament associated protein kinase activities from bovine spinal cord. Cell and Molecular Neurobiology 10, 369-82.

FRIEDE, R. L. \& SAMORAJSKI, T. (1970) Axon caliber related to neurofilaments and microtubules in sciatic nerve fibres of rats and mice. Anatomical Record 167, 379-86.

GOLD, B. G., PRICE, D. L., GRIFFIN, J. W., ROSENFELD, J., HOFFMAN, P. N., STERNBERGER, N. H. \& STERNBERGER, L. A. (1988) Neurofilament antigens in acrylamide neuropathy. Journal of Neuropathology and Experimental Neurology $47,145-57$.

GOLDSTEIN, M. E., STERNBERGER, N. H. \& STERNBERGER, L. A. (1987) Phosphorylation protects neurofilaments against proteolysis. Journal of Neuroimmunology 14, 149-60.

GONDA, Y., NISHIZAWA, K., ANDOS, S., KITAMURA, Y., MINOURA, NISHI, Y. \& INAGAKI, M. (1990) Involvement of protein kinase $C$ in the regulation of assemblydisassembly of neurofilaments in vitro. Biochemical and Biophysical Research Communications 167, 1316-25.

GRIFFIN, J. W. \& WATSON, D. F. (1988) Axonal transport in neurological disease. Annals of Neurology 23, 3-13.

GRIFFIN, J. W. , FAHENSTOCK, K. E., PRICE, D. L. \& CORK, L. C. (1983) Cytoskeletal disorganization induced by local application of beta, beta'-iminodipropionitrile and 2,5hexanedione. Annals of Neurology 14, 55-61.

HART, C. E., NUCKOLLS, G. H. \& WOOD, J. G. (1987) Subcellular compartmentalization of phosphorylated neurofilament polypeptides in neurons. Cell Motility and the Cytoskeleton 7, 393-403.
1987; Pant, 1988) so that dephosphorylation at specific sites may allow degradation in situ by endogenous proteases.

Investigation of animal model systems with altered NF structure should allow some insight into the role of phosphorylation at the node in vivo.

\section{Acknowledgements}

We thank Diane Alessi for assistance in electron microscopy, and Brenda Johnson and Subhash Datta for assistance in biochemistry. This work was supported by VA Merit Review grants (MM and DJF) and the NIH (NINDS NS 27771-3, DJF). This work was presented in abstract form at the Annual Meeting of the Society for Neuroscience in St. Louis, MO in October 1990.
HISANAGA, S.-I. \& HIROKAWA, N. (1989) The effects of dephosphorylation on the structure of the projections of neurofilaments. Journal of Neuroscience 9, 959-66.

HiSANAGA, S.-I. \& HiRokAWA, N. (1990) Dephosphorylation-induced interactions of neurofilaments with microtubules. Journal of Biological Chemistry 265, 21852-8.

HOFFMAN, P. N. \& LASEK, R. J. (1975) The slow component of axonal transport: identification of major structural polypeptides of the axon and their generality among mammalian neurons. Journal of Cell Biology 66, 351-66.

HOFFMAN, P. N., GRIFFIN, J. W. \& PRICE, D. L. (1984) Control of axonal caliber by neurofilament transport. Journal of Cell Biology 9, 705-14.

HOFFMAN, P. N., THOMPSON, G. W., GRIFFIN, J. W. \& PRICE, D. L. (1985) Changes in neurofilament transport coincide temporally with alterations in the caliber of axons in regenerating motor fibres. Journal of Cell Biology 101, 1332-40.

JULIEN, J.-P. \& MUSHYNSKI, W. E. (1982) Multiple phosphorylation sites in mammalian neurofilament polypeptides. Journal of Biological Chemistry 257, 10467-70.

JULIEN, J.-P. I-MUSHYNSKI, W. E. (1983) The distribution of phosphorylation sites among identified proteolytic fragments of mammalian neurofilaments. Journal of Biological Chemistry 2558, 4019-25.

JULIEN, J.-P., SMOLUK, G. D. \& MUSHYNSKI, W. E. (1983) Characteristics of the protein kinase activity associated with rat neurofilament preparations. Biochemica et Biophysica Acta 755, 25-31.

KARLSSC N, J.-E., ROSENGREN, L. E. \& HAGLIN, K. G. (1989) Polyclonal antisera to the individual neurofilament triplet proteins: a characterization using ELISA and immunoblotting Journal of Neurochemistry 53, 759-65.

LEE, V. M.-Y., CARDEN., M. J., SCHLAEFER, W. W. \& TROJANOWSKI, J. Q. (1987) Monoclonal antibodies distinguish several differentially phosphorylated states of the two largest rat neurofilament subunits (NF-H and 
NF-M) and demonstrate their existence in the normal nervous system of adult rats. Journal of Neuroscience 7, 3474-88.

LEWIS, S. E. \& NIXON, R. A. (1988) Multiple phosphorylated variants of the high molecular mass subunit of neurofilaments in axons of retinal cell neurons: characterization and evidence for their differential association with stationary and moving neurofilaments. Journal of Cell Biology 107, 2689-701.

MATA, M., ALESSI, D. \& FINK, D. J. (1990) S100 is preferentially distributed in myelin-forming Schwann cells. Journal of Neurocytology 19, 432-42.

MINAMI, Y. \& SAKAI, H. (1985) Dephosphorylation suppresses the activity of neurofilament to promote tubulin polymerization. FEBS Letters 185, 239-42.

MORI, H. \& KUROKAWA, M. (1980) Morphological and biochemical characterization of neurofilaments isolated from the rat peripheral nerve. Biomedical Research 1, 24-31.

NIXON, R. A. \& LEWIS, S. E. (1986) Differential turnover of phosphate groups on neurofilament subunits in mammalian neurons in vivo. Journal of Biological Chemistry 261, 16298-301.

NIXON, R. A., LEWIS, S. E. \& MAROTTA, C. A. (1987) Posttranslational modification of neurofilament proteins by phosphate during axoplasmic transport in retinal ganglion cell neurons. Journal of Neuroscience 7, 1145-58.

PANT, H. C. (1988) Dephosphorylation of neurofilament proteins enhances their susceptibility to degradation by calpain. Biochemical Joumal 256, 665-8.

PARHAD, I. M., GRIFFIN, J. W., CLARK, A. W. \& KOVES, J. F. (1984) Doxorubicin intoxication: neurofilamentous axonal changes with subacute neuronal death. Journal of Neuropathology and Experimental Neurology 43, 188-200.

PESTRONK, A., WATSON, D. F. \& YUAN, C. M. (1990) Neurofilament phosphorylation in peripheral nerve: changes with axonal length and growth state. Journal of Neurochemistry 54, 977-82.

ROSENFELD, J., DORMAN, M. E., GRIFFIN, J. W., STERNBERGER, L. A., STERNBERGER, N. H. \& PRICE, D. L. (1987) Distribution of neurofilament antigens after axonal injury. Journal of Neuropathology and Experimental Neurology $46,269-82$.

SHAW, G. \& WEBER, K. (1982) Differential expression of neurofilament triplet proteins in brain development. Nature 298, 277-9.

SHAW, G., OSBORN, M. \& WEBER, K. (1986) Reactivity of a panel of neurofilament antibodies on phosphorylated and dephosphorylated neurofilaments. European Journal of Cell Biology 42, 1-9.

SIEGEL, G. J., DESMOND, T. \& ERNST, S. A. (1986) Immunoreactivity and ouabain-dependent phosphorylation of $\left(\mathrm{Na}^{+}+\mathrm{K}^{+}\right)$-adenosine triphosphatase catalytic subunit doublets. Journal of Biological Chemistry 261, 13768-76.

SIHAG, R. K. \& NIXON, R. A. (1989) In vivo phosphorylation of distinct domains of the 70-kilodalton neurofilament subunit involves different protein kinases. Journal of Biological Chemistry 264, 457-64.

STERnBERGER, L. A. \& STERnBERGER, N. H. (1983) Monoclonal antibodies distinguish phosphorylated and nonphosphorylated forms of neurofilaments in situ. Proceedings of the National Academy of Sciences (USA) 80, 6126-30.

STERNBERGER, L. A., HARWELL, L. W. \& STERNBERGER, N. H. (1982) Neurotypy: regional individuality in rat brain detected by immunocytochemistry with monoclonal antibodies. Proceedings of the National Academy of Sciences (USA) 79, 1326-30.

SZARO, B. G., WHITNALL, M. H. \& GAINER, H. (1990) Phosphorylation-dependent epitopes on neurofilament proteins and neurofilament densities differ in axons in the corticospinal and primary sensory dorsal column tracts in the rat spinal cord. Journal of Comparative Neurology 302, 220-35.

TOWBIN, H., STAEHELIN, T. \& GORDON, J. (1979) Electrophoretic transfer of proteins from polyacrylamide gels to nitrocellulose sheets: procedure and some applications. Proceedings of the National Academy of Sciences (USA) 76, $4350-4$

WATSON, D. F. \& GRIFFIN, J. W. (1987) Vacor neuropathy: ultrastructural and axonal transport studies. Journal of Neuropathology and Experimental Neurology 46, 96-108.

WATSON, D. F., GRIFFIN, J. W., FITTRO, K. P. \& HOFFMAN, P. N. (1989) Phosphorylated-dependent immunoreactivity of neurofilaments increases during axonal maturation and beta, beta-iminodiproprionitrile intoxication. Journal of Neurochemistry 53, 1818-29.

WILKERSON, L. (1990) SYSTAT: The System for Statistics. Evanston, IL: SYSTAT, Inc.

WILLARD, M. \& SIMON, C. (1983) Modulation of neurofilament axonal transport during the development of rabbit retinal ganglion cells. Cell 35, 551-9.

WONG, J., HUTCHISON, S. B. \& LIEM, R. K. H. (1984) An isoelectric variant of the 150,000 -dalton neurofilament. Journal of Biological Chemistry 259, 10867-74. 